\title{
Large-scale collective motion of RFGC galaxies in curved space-time
}

\author{
S. L. Parnovsky ${ }^{1 \star}$ and A. S. Parnowski ${ }^{2}$ \\ ${ }^{1}$ Astronomical Observatory of Kyiv Taras Shevchenko National University, Observatorna str., 3, 04058, Kyiv, Ukraine \\ ${ }^{2}$ Space Research Institute of NASU \& NSAU, prosp. Akad. Glushkova, 40, korp. 4/1, 03680 MSP, Kyiv-187, Ukraine
}

Accepted . Received ; in original form 4 June 2017

\begin{abstract}
We consider large-scale collective motion of flat edge-on spiral galaxies from the Revised Flat Galaxy Catalogue (RFGC) taking into account the curvature of space-time in the Local Universe at the scale $100 h^{-1} \mathrm{Mpc}$. We analyse how the relativistic model of collective motion should be modified to provide the best possible values of parameters, the effects that impact these parameters and ways to mitigate them. Evolution of galactic diameters, selection effects, and difference between isophotal and angular diameter distances are inadequate to explain this impact. At the same time, measurement error in $\mathrm{HI}$ line widths and angular diameters can easily provide such an impact. This is illustrated in a toy model, which allows analytical consideration, and then in the full model using Monte Carlo simulations. The resulting velocity field is very close to that provided by the non-relativistic model of motion. The obtained bulk flow velocity is consistent with $\Lambda$ CDM cosmology.
\end{abstract}

Key words: galaxies: kinematics and dynamics - galaxies: distances and redshifts - galaxies: spiral - relativity - methods: numerical

\section{INTRODUCTION}

At present time the Universe is essentially inhomogeneous on the scales of about 10-100 Mpc. The development of initial fluctuations led to an observable large-scale structure. The regions with increased matter density provide an additional attraction of surrounding galaxies. The regions with decreased density, e.g. voids, also make an input to the collective large-scale motion of galaxies on the background of Hubble expansion. Investigation of such motion on one side allows to map the matter density, including dark ' matter, in the Local Universe, and on the other side its parameters are linked with cosmological parameters. All of this makes the study of collective galaxy motions important.

In recent years a number of articles was published claiming that typical velocities of large-scale collective motions are inconsistent with the standard $\Lambda$ CDM model. For example, Watkins et al. (2009) obtained the value $407 \pm 81 \mathrm{~km} \mathrm{~s}^{-1}$ at the scale $100 h^{-1} \mathrm{Mpc}$, whereas the $\Lambda \mathrm{CDM}$ model gives about $250 \mathrm{~km} \mathrm{~s}^{-1}$. However, our estimation of $210 \pm 86 \mathrm{~km} \mathrm{~s}^{-1}$ at the same scale, obtained in the article (Parnovsky \& Parnowski 2010), is consistent with the $\Lambda \mathrm{CDM}$ predictions. Additionally, in the same article we obtained from the peculiar velocities the constraints on the cosmological parameters $\Omega_{m}$ and $\sigma_{8}$ and their combinations, which match the other more precise constraints like baryonic acoustic oscillations or WMAP observations.

In the article (Parnovsky \& Parnowski 2010) we used a sample of RFGC galaxies with measured redshifts and $\mathrm{HI}$ line widths. The Revised Flat Galaxy Catalogue (RFGC) Karachentsev et al.

\footnotetext{
^ E-mail: par@observ.univ.kiev.ua
}

1999) and its previous version Flat Galaxy Catalogue (FGC) (Karachentsev et al. 1993) contain the information about Right Ascension and Declination for the epochs J2000.0 and B1950.0, galactic longitude and latitude, major and minor blue and red diameters in arcminutes in the POSS-I diameter system, morphological type of the spiral galaxies according to the Hubble classification, index of the mean surface brightness and some other parameters, which are not used in this article. The RFGC contains data about 4236 flat edge-on spiral galaxies, almost uniformly covering the celestial sphere and satisfying the conditions $a_{b} / b_{b} \geqslant 7$ and $a_{b}>0$. 6 . Here $a_{b}$ and $b_{b}$ are the major and minor axial diameters in the $a_{25}$ system. The original goal of this catalogue was to estimate the distance to galaxies according to the Tully-Fisher relation in the "H I line width - linear diameter" version without using their redshifts. The data about the redshifts and H I line widths or gas rotation velocities $V_{\text {rot }}$ were taken from different sources. There were a number of gradually increasing samples of galaxies with such data (Karachentsev et al. 2000; Parnovsky et al. 2001; Parnovsky \& Tugav 2004). The latest version of this sample including 1623 galaxies was compiled and described by Parnovsky \& Parnowski (2010). A list of peculiar velocities based upon this list in the non-relativistic model of motion was assembled by Parnovsky \& Parnowski (2009).

In this article we use the same sample, but with different model of collective motion of galaxies (Kudrya \& Alexandrov 2002, 2004), based upon the general theory of relativity (GTR). This model was applied earlier to the previous version of the sample by Parnovsky \& Gaydamaka (2004); however, the present article offers a much more in-depth analysis. 


\section{DESCRIPTION OF MODELS OF COLLECTIVE MOTION OF GALAXIES}

We assume that there is a three-dimensional velocity field of collective galactic motions on the background of Hubble expansion. We consider the galaxies in the sample not as massive objects, but rather as test particles, whose peculiar velocities are indicators of the large-scale velocity field. Using the multipole decomposition of large-scale velocity field up to quadratic terms in distance and switching to the radial component we obtain the expression for radial velocity of an individual galaxy. The actually measured radial velocities differ from those predicted by this model due to deviations from the statistical Tully-Fisher relation, influence of motions with scales much less than the sample depth (fall towards nearby attractors) and random errors. Treating these errors as stochastic we can use the least squares method to calculate the parameters of the model.

\subsection{Non-relativistic model of collective motion}

Before discussing the relativistic model of collective motion, let us briefly recall the non-relativistic models, introduced in the article (Parnovsky et al. 2001) and used by Parnovsky \& Tugay (2004) and Parnovsky \& Parnowski (2010). We will start from the more complex of them, namely the DQO-model.

$V=R+V^{d i p}+V^{q u a}+V^{o c t}+\delta V$.

Here $V$ is a radial velocity of the galaxy in the CMB isotropy frame, $R=H r$ is the Hubble velocity, $r$ is the distance to the galaxy, $\delta V$ is a random error, $V^{d i p}, V^{q u a}$ and $V^{o c t}$ are the dipole (D), quadrupole $(\mathrm{Q})$ and octopole $(\mathrm{O})$ components of the non-Hubble cosmic flow. They are given by the following expressions:

$$
\begin{aligned}
V^{d i p}= & D_{i} n_{i}, \\
V^{q u a}= & R Q_{i k} n_{i} n_{k}=R\left(q_{1}\left(n_{1}^{2}-n_{3}^{2}\right)+q_{2}\left(n_{2}^{2}-n_{3}^{2}\right)\right. \\
& \left.+q_{3} n_{1} n_{2}+q_{4} n_{1} n_{3}+q_{5} n_{2} n_{3}\right) \\
V^{\text {oct }}= & R^{2}\left(O_{i k l} n_{i} n_{k} n_{l}+P_{i} n_{i}\right)=R^{2}\left(P_{i} n_{i}\right. \\
& +o_{1}\left(3 n_{1} n_{2}^{2}-n_{1}^{3}\right)+o_{2}\left(3 n_{1} n_{3}^{2}-n_{1}^{3}\right) \\
& +o_{3}\left(3 n_{2} n_{1}^{2}-n_{2}^{3}\right)+o_{4}\left(3 n_{2} n_{3}^{2}-n_{2}^{3}\right) \\
& +o_{5}\left(3 n_{3} n_{1}^{2}-n_{3}^{3}\right)+o_{6}\left(3 n_{3} n_{2}^{2}-n_{3}^{3}\right) \\
& \left.+o_{7} n_{1} n_{2} n_{3}\right) .
\end{aligned}
$$

Here we use the Einstein rule - summation by repeated indices; $n_{i}$ are Cartesian components of the unit vector $\boldsymbol{n}$ towards the galaxy, connected with the galactic coordinates $l$ and $b$ in the following way:

$$
\begin{aligned}
& n_{1}=n_{z}=\sin b, \\
& n_{2}=n_{x}=\cos l \cos b, \\
& n_{3}=n_{y}=\sin l \cos b .
\end{aligned}
$$

The dipole component (bulk motion) is described by the vector $\boldsymbol{D}$. The quadrupole component is described by the symmetrical traceless tensor $\mathbf{Q}$. It has 5 independent parameters $q_{i}$. The octopole component can be described by one rank 3 tensor, but we divide it into a trace characterized by vector $\boldsymbol{P}$ and a tensor $\mathbf{O}$, which is antisymmetrical with respect to each pair of indices. The latter has 7 independent parameters $o_{i}$.

Hubble velocity is determined from the generalized TullyFisher relation in the "angular diameter - H I line width" version by the following formula

$$
\begin{aligned}
R= & \left(C_{1}+C_{2} B+C_{3} B T+C_{4} U\right) \frac{W}{a} \\
& +C_{5}\left(\frac{W}{a}\right)^{2}+C_{6} \frac{1}{a},
\end{aligned}
$$

where $W$ is a corrected $\mathrm{H}$ I line width in $\mathrm{km} \mathrm{s}^{-1}$ measured at 50 per cent of the maximum, $a$ is a corrected major galaxies' angular diameter in arcminutes on red POSS and ESO/SERC reproductions, $U$ is a ratio of major galaxies' angular diameters on red and blue reproductions, $T$ is a morphological type indicator $\left(T=I_{t}-5.35\right.$, where $I_{t}$ is a Hubble type; $I_{t}=5$ corresponds to type $\mathrm{Sc}$ ), and $B$ is a surface brightness indicator $\left(B=I_{S B}-2\right.$, where $I_{S B}$ is a surface brightness index from RFGC; brightness decreases from I to IV). Note that the statistical significance of each term in eq. (6) is greater than 99 per cent according to the F-test (Fisher 1954; Hudson 1964).

Thus, the DQO-model contains 24 parameters, namely 3 components of the vector $D, 6$ coefficients $C_{i}, 5$ parameters $q_{i}$ of the tensor $\mathbf{Q}, 3$ components $p_{i}$ of the vector $\boldsymbol{P}$ and 7 parameters $o_{i}$ of the tensor $\mathbf{O}$. By rejecting $V^{\text {oct }}$ we get a simpler DQ-model with 14 components. Further rejecting $V^{q u a}$ leads to the simplest D-model with 9 components.

\subsection{Relativistic model of collective motion}

The existence of multipole components is due to density inhomogeneities in the Universe. For homogeneous isotropic Universe instead of equation (1) we would have the simple Hubble law $V=H r$. Hubble expansion is due to cosmology and thus is most adequately described in the framework of GTR. Such a description raises a problem of distance measurement. The main types of distances used are the redshift distance, photometric distance and angular diameter distance. They are related to each other through formulae containing cosmological parameters. In our case the natural choice is the angular diameter distance, since we determine distances by the generalized Tully-Fisher relation using angular diameters. Thus, when speaking about distance $r$ in relativistic models, we will mean angular diameter distance.

For the homogeneous isotropic cosmological models the dependence of the velocity $V=c z$ on $R=H r$ in the next order in $R$ has the form

$V=R+\gamma R^{2}$.

The coefficient $\gamma$ is expressed through the deceleration parameter $q$ by

$\gamma=\frac{3+q}{2 c}$.

For different types of distance another expression for $\gamma$ should be used. For the standard $\Lambda \mathrm{CDM}$ cosmology we have

$q=\frac{\Omega_{m}}{2}-\Omega_{\Lambda}=-0.61$,

where $\Omega_{m}$ and $\Omega_{\Lambda}$ are relative densities of matter, including dark matter, and dark energy respectively. Numerical estimations are based on the results of 7-year WMAP observations (Komatsu et al. 2010). Introducing (9) into (8) we obtain

$\gamma_{0}=3.98 \cdot 10^{-6} \mathrm{~s} \mathrm{~km}^{-1}$.

Now let us consider a relativistic model of galaxy motion in inhomogeneous space-time. It was developed by Kudrya \& Alexandrov (2002, 2004). Instead of (1) we use the equation

$V_{\text {rel }}=R+V^{\text {dip }}+V^{q u a}+V^{o c t}+\gamma R^{2}+\delta V$.

Here $V_{\text {rel }}$ is still given in the CMB frame, the expressions for the dipole (2) and qudrupole (3) components remain the same, and the octopole component assumes the form 
$V_{r e l}^{\text {oct }}=R^{2}\left(P_{i} n_{i}+O_{i j k} n_{i} n_{j} n_{k}+S_{i j} n_{i} n_{j}\right)$

Here $\mathbf{S}$ is a symmetric traceless tensor, characterized by 5 independent parameters $s_{i}$ :

$$
\begin{aligned}
S_{i j} n_{i} n_{j}= & s_{1}\left(n_{1}^{2}-n_{3}^{2}\right)+s_{2}\left(n_{2}^{2}-n_{3}^{2}\right) \\
& +s_{3} n_{1} n_{2}+s_{4} n_{1} n_{3}+s_{5} n_{2} n_{3} .
\end{aligned}
$$

The parameter $\gamma$ is related to the deceleration parameter by

$$
\gamma=\frac{3+q}{2 c}+\frac{1}{3 c} Q_{i k} Q^{i k} \text {. }
$$

This equation reduces to equation (8) in absence of quadrupole component, for example, for homogeneous models. Similarly to the non-relativistic case, we can reduce the relativistic DQO-model to DQ- and D-models by rejecting highest-order multipoles.

In the papers (Kudrya \& Alexandrov 2002, 2004) it was shown that the relation

$$
\begin{aligned}
C_{\alpha \beta \gamma \delta} V^{\beta} V^{\delta}= & \frac{H^{2}}{c^{2}}\left(-2 c S_{\alpha \gamma}+6 Q_{\alpha \gamma}-3 Q_{\alpha}^{\epsilon} Q_{\epsilon \gamma}\right. \\
& \left.-\left(V_{\alpha} V_{\gamma}-g_{\alpha \gamma} Q_{\epsilon \eta} Q^{\epsilon \eta}\right)\right)
\end{aligned}
$$

must hold. Here Greek indices denote four-dimensional components, $g_{\alpha \beta}$ is a metrical tensor, and $C_{\alpha \beta \gamma \delta}$ is a Weyl tensor. The spatial parts of four-dimensional tensors $Q_{\alpha \beta}$ and $S_{\alpha \beta}$ coincide with three-dimensional tensors $\mathbf{Q}$ and $\mathbf{S}$, and their temporal and mixed parts are much smaller. The four-dimensional velocity vector $V_{\alpha}$ can be assumed equal to $\left(g_{00}^{-1 / 2}, 0,0,0\right)$.

Now all that remains is to supply the relativistic model with an expression for the angular diameter distance. Since we consider the terms proportional to $R^{2}$ separately, we should remove the terms quadratic in distance from the generalised Tully-Fisher relation (6):

$R=\left(C_{1}+C_{2} B+C_{3} B T+C_{4} U\right) \frac{W}{a}+C_{5} \frac{1}{a}$.

Note that all the remaining terms in this equation are inverse proportional to the angular diameter $a$.

Using the data about radial velocities, H I line widths, angular diameters, morphological types, surface brightness indices and axial ratio of galaxies it is possible to obtain the value and errors for all 29 parameters of the relativistic model. For the previous version of the sample it was done by Parnovsky \& Gaydamaka (2004). The statistical weights of all galaxies are assumed to be the same. Besides the whole sample we also use subsamples limited in depth $R<R_{\max }$. They are defined as follows: they contain all galaxies, which have Hubble velocity less than $R_{\max }$ in the non-relativistic D-model. The results of processing of the subsamples with $R_{\max }=8000 \mathrm{~km} \mathrm{~s}^{-1}$ and $R_{\max }=10000 \mathrm{~km} \mathrm{~s}^{-1}$ are presented in Table 1 It contains information about the standard deviation $\sigma$, coefficients of the Tully-Fisher relation, apex coordinates and modulus of the bulk flow and parameters of the multipole components. The results of processing of the same subsamples in the non-relativistic model are given in the paper (Parnovsky \& Parnowski 2010).

\subsection{Semirelativistic model of collective motion}

The obtained results appear to have problems - the values of $\mathbf{S}$ are 200 times larger then their estimation from equation 15 . The same situation appeared when processing the previous sample. It is easy to see that the right-hand part of equation 15 is dominated by the term containing tensor $\mathbf{S}$ due to the speed of light.

For the homogeneous isotropic Universe the Weyl tensor and tensors $\mathbf{S}$ and $\mathbf{Q}$ vanish. They are connected with spatial inhomogeneities of density distribution, e.g. attractors and voids. The left part of this relation is a sum of inputs of individual inhomogeneities. For a spherically symmetric attractor with an excessive mass $M$ at a distance $u$ the spatial part of the tensor $C_{\alpha \beta \gamma \delta} V^{\beta} V^{\delta}$ after reduction to eigenaxes receives the form:

$C_{\alpha \beta \gamma \delta} V^{\beta} V^{\delta}=\frac{G M}{c^{2} u^{3}}\left(\begin{array}{ccc}2 & 0 & 0 \\ 0 & -1 & 0 \\ 0 & 0 & -1\end{array}\right)$.

This value falls cubically with distance, so the main input is provided by nearby attractors. In the paper (Parnovsky \& Gaydamaka 2004) the input of the Great Attractor, Perseum-Pisces superclaster, Shepley concentration and Virgo cluster were analyzed. The excessive masses and distances to attractors were taken from the paper (Marinoni et al. 1998). It was shown that the greatest input is provided by the Virgo cluster. Adding the inputs of all attractors we obtain an estimation of tensor S, which appears to be 200 times smaller than the calculated values. This is caused by the same distribution of the tensors $\mathbf{Q}$ and $\mathbf{S}$ over the celestial sphere. Due to measurement errors and deviations from the Tully-Fisher relation the tensor $\mathbf{S}$ "borrows" some of the value of tensor $\mathbf{Q}$. Unfortunately, with the quality and quantity of available observational data we are unable to correctly separate the inputs of these tensors. Thus, taking into account the small values of tensor $\mathbf{S}$, a so-called semirelativistic model was introduced in the paper (Parnovsky \& Gaydamaka 2004), which differs from the full relativistic model only by the dropped term with tensor S. So, it will be possible to use the relativistic model only when we get samples with significantly better quality and larger depth. For this reason, we switch to the semirelativistic model 2, 3, 4, 11, 16. The results of processing in semirelativistic model are also given in Table 1 The semirelativistic DQ- and D-models are exactly the same as their relativistic counterparts.

However, in both the relativistic and semirelativistic models there is a serious problem. The calculated value of $\gamma$ appears to be way off the expected value, namely $(-14.1 \pm 2.9) \cdot 10^{-6} \mathrm{~s} \mathrm{~km}^{-1}$ in the relativistic model and $(-16.6 \pm 2.6) \cdot 10^{-6} \mathrm{~s} \mathrm{~km}^{-1}$ in the semirelativistic model for $R_{\max }=10000 \mathrm{~km} \mathrm{~s}^{-1}$. This value essentially differs from $\gamma_{0}=3.98 \cdot 10^{-6} \mathrm{~s} \mathrm{~km}^{-1}$ (10) calculated from cosmological parameters. As one can see from Table 1 the second term in equation (14) is negligible and cannot be responsible for the discussed effect. Naturally, we do not question the values of the cosmological parameters and the reason should be sought elsewhere.

In the next two section we consider the reasons, which could lead to the deviation of the calculated value of $\gamma$ from its value (10) obtained from the cosmological parameters. These reasons can be caused either by the dependence of linear galaxy diameters on distance or by the influence of measurement errors.

\section{SHIFT OF $\gamma$ DUE TO DEPENDENCE OF LINEAR GALAXY DIAMETERS ON DISTANCE}

When determining the distances to the galaxies using the generalised Tully-Fisher relation in the "linear diameter - H I line width" version, we assume that galaxies with the same $\mathrm{H}$ I line width, morphological type, axial ratio and surface brightness index have the same linear diameter $L$. Let us consider the opposite case when the linear diameter weakly depends on the distance $r$ according to the law

$L(r)=L_{0}+r \frac{d L}{d r}$ 
Table 1. Parameters of the relativistic (R), semirelativistic (SR) and semirelativistic with fixed $\gamma(\mathrm{SR} \gamma)$ models of collective motion for the subsamples with $R_{\max }=10000 \mathrm{~km} \mathrm{~s}^{-1}$ and $R_{\max }=8000 \mathrm{~km} \mathrm{~s}^{-1}$

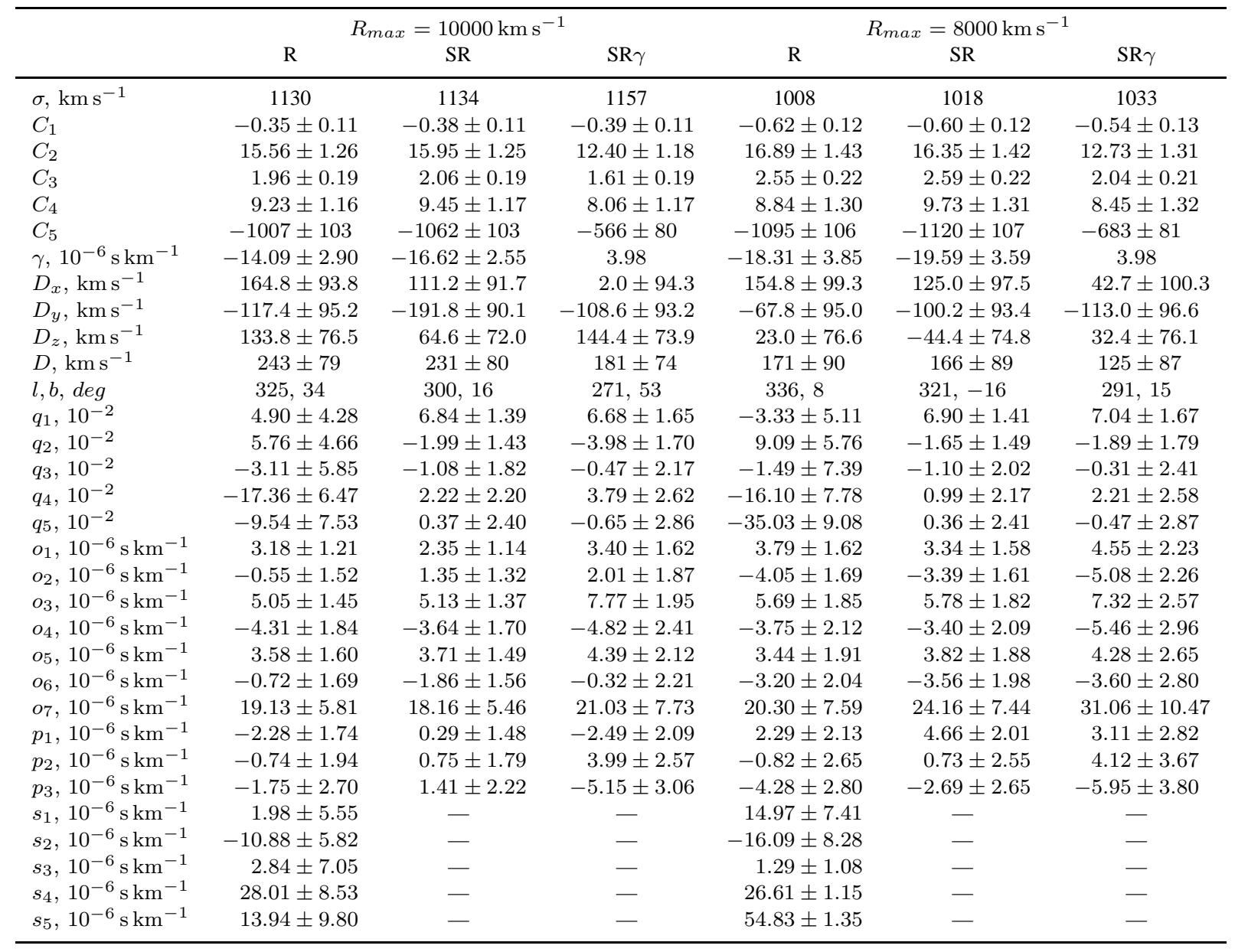

Here $L_{0}$ is the linear diameter of nearby galaxies according to Tully-Fisher relation. On the other hand, if we express the angular diameters $a$ in radians, the linear diameters will be given by

$$
L(r)=a r \text {. }
$$

This yields a problem: we determine the distances to the galaxies using the Tully-Fisher relation in an assumption that the linear diameters of the galaxies are equal to $L_{0}$ rather than $L(r)$. Thus, the apparent distance $\rho$ will be equal to

$\rho=\frac{L_{0}}{a}$.

Combining the latter two formulae we can express the true distance $r$ through the apparent distance $\rho$ :

$r=\rho\left(1+\frac{r}{L_{0}} \frac{d L}{d r}\right)$.

Let us calculate the shift of $\gamma$ due to this effect. Radial velocities of galaxies according to equation (7) are given by

$V=H r+\gamma_{0}(H r)^{2}$,

where $\gamma_{0}$ is the true value of $\gamma$. However, instead of this formula we use the following expression:

$V=H \rho+\gamma(H \rho)^{2}$.

It is trivial to find that

$$
\Delta \gamma=\gamma-\gamma_{0}=\frac{1}{H L_{0}} \frac{d L}{d r}
$$

There are several effects leading to the dependence (24). Let us consider them one by one.

\subsection{Effect of galaxy evolution}

Let us assume that galaxies evolve with time changing their linear diameters with a characteristic rate $\dot{L}=d L / d t$. Since we observe more distant galaxies at earlier stages of development, we can write

$\frac{d L}{d r}=-\frac{\dot{L}}{c}, \Delta \gamma=-\frac{1}{H c} \frac{\dot{L}}{L}$

This effect is described, in particular, by Weinberg (1972).

The question is, whether it alone can explain the observed value of $\Delta \gamma \sim 2.2 \cdot 10^{-5} \mathrm{~s} \mathrm{~km}^{-1}$ ? A simple estimation shows that would this be the case, the galaxies should shrink with a typical rate of $4.7 \cdot 10^{-10} \mathrm{yr}^{-1}$. This value contradicts to our knowledge of galaxy evolution. In particular, such large shrinking rate would lead to decrease of galaxies by about 12 per cent in one orbital period of the Sun in the Milky Way. Thus, this effect cannot make an essential contribution to the observed shift of $\gamma$. 


\subsection{Effect of selection by angular diameters}

The Tully-Fisher relation is statistical. In fact, galaxies can be smaller or larger than given by it. For galaxies with angular diameters close to the threshold $a_{b}=0.6$ there is a selection. Large galaxies will enter RFGC and the small ones will have too small angular diameters and will be rejected. Thus, the average linear diameters of RFGC galaxies will increase with distance. This is usually referred to as Malmquist bias. According to equation (24), these effects will lead to a positive shift of $\Delta \gamma$. This shift has the opposite sign to the observed one and, therefore, cannot be its cause. Nevertheless, this effect cannot be totally neglected and further we will estimate its value using Monte Carlo simulations.

\subsection{Effect of cosmological decrease of observed surface brightness of galaxies}

Consideration of space-time curvature even in the simplest homogeneous isotropic cosmological models leads to a number of effects. One of these effects yields the decrease of observed surface brightness of galaxies. It is quite evident that the surface brightness is proportional to $(r / D)^{2}$, where $\mathrm{D}$ is the photometric distance. In flat space-time there is no difference between $r$ and $D$ and the surface brightness does not depend on the distance. In curved spacetime at small $z$ we can use the expressions for $r$ and $D$ from the book (Zeldovich \& Novikov 1983), which yield $I=I_{N R}(1-4 z)$. Here $I_{N R}$ is the surface brightness in the flat space-time and $z$ is the redshift. This expression is a low-z limit of the Tolman effect (Tolman 1930, 1934), which describes the decrease of surface brightness by a factor of $(1+z)^{4}$. Switching to the brightness $\mu=-2.5 \lg I$ measured in mag $/ \square^{\prime \prime}$ we obtain the shift

$$
\begin{aligned}
\Delta \mu= & \mu-\mu_{N R}=\frac{10}{\ln 10} R / c \\
& =1.45 \cdot 10^{-5} R \mathrm{mag} / \square^{\prime \prime} \mathrm{s} \mathrm{km}^{-1} .
\end{aligned}
$$

For a galaxy at the distance $100 h^{-1} \mathrm{Mpc}$ this gives $\Delta \mu=$ $0.145 \mathrm{mag} / \square^{\prime \prime}$. Earlier such effects were considered, e.g. by Sandage \& Lubin (2001).

If galaxies had sharp edges where the surface brightness instantly vanishes, this effect would be of no interest for us. However, for real galaxies the surface brightness gradually falls to the edges according to the de Vaucouleurs law $I(l)=I_{0} e^{-l / l_{0}}, \mu(l)=$ $\mu_{0}+1.0857 l / l_{0}$ (de Vaucouleurs 1959). Here $I_{0}$ and $\mu_{0}$ are the values of $I$ and $\mu$ in the centre of the galaxy, $l$ is the radial distance from the centre of galaxy and $l_{0}$ is a characteristic radial scale. For spiral galaxies the scale $l_{0}$ weakly depends on the morphological type. For bright, comparable to the Milky Way, galaxies it ranges from 1 to $10 \mathrm{kpc}$ (Reshetnikov 2001). A more accurate estimation was obtained by Fathi et al. (2010) using the sample of more than 30000 galaxies. The average value of $l_{0}$ appeared to be equal to $3.8 \pm 2.1 \mathrm{kpc}$. For smaller galaxies with the total stellar mass $10^{9}-10^{10} M_{\odot}$ they estimated it as $1.5 \pm 0.7 \mathrm{kpc}$ and for larger galaxies with the total stellar mass $10^{11}-10^{12} M_{\odot}-$ as $5.7 \pm 1.9 \mathrm{kpc}$. For the later types of galaxies, which are predominant in RFGC, the value of $l_{0}$ does not typically exceed $2 \mathrm{kpc}$, according to Freeman (1970). In his sample of 36 galaxies the maximum value of $l_{0}$ was $6.1 \mathrm{kpc}$, and the second largest was $4.5 \mathrm{kpc}$.

The linear diameters of RFGC galaxies are determined at the isophotal level $\mu=25 \mathrm{mag} / \square^{\prime \prime}$. Due to the abovementioned effect for distant galaxies this boundary is shifted with respect to its position in flat space-time. For instance, for a galaxy at $r=100 h^{-1} \mathrm{Mpc}$ the region corresponding to the isophotal level
$25 \mathrm{mag} / \square^{\prime \prime}$ in the flat space-time would appear at the isophotal level $25.145 \mathrm{mag} / \square^{\prime \prime}$ in the real Universe.

For this reason, the isophotal boundaries will be shifted towards the centre of the galaxy by $\delta l=1.33 \cdot 10^{-5} l_{0} R \mathrm{~s} \mathrm{~km}^{-1}$. Naturally, the apparent decrease of isophotal diameter $\delta L$ will be twice this value. Using this distance-dependent isophotal diameter instead of constant linear diameter, we obtain the so-called isophotal distance instead of the angular diameter distance.

The expression (8) is derived for angular distance only. In reality, however, we deal with isophotal distances with a slightly different value of $\gamma$. From the equations (26) and $L(r)=L_{0}-\delta L$ we get $\delta \gamma=-2.66 \cdot 10^{-5} l_{0} / L_{0} \mathrm{~s} \mathrm{~km}^{-1}$. Let us estimate this value. As a typical galaxy diameter we take the value for the Milky Way: $L_{0}=30 \mathrm{kpc}$. The distribution of $\lg L_{0}$ for RFGC galaxies, where $L_{0}$ is expressed in kpc, is given by Kudrya et al. (1997). The maximum of this distribution corresponds to the interval from 1.3 to 1.4 , ehich corresponds to $L_{0} \sim 22 \mathrm{kpc}$. The mean value of $L_{0}$ for RFGC galaxies should be taken slightly larger because $\left\langle L_{0}\right\rangle>10^{\left\langle\lg L_{0}\right\rangle}$. With $L_{0}=30 \mathrm{kpc}$ and $l_{0}=3 \div 5 \mathrm{kpc}$ we get $\Delta \gamma=(-2.7 \div-4.4) \cdot 10^{-6} \mathrm{~s} \mathrm{~km}^{-1}$, which constitutes from 12 to 20 per cent of observed shift. Even the extreme estimation with $L_{0}=22 \mathrm{kpc}, l_{0}=10 \mathrm{kpc}$ can explain only 50 per cent of the observed shift. For edge-on spiral galaxies the surface photometry was performed in a series of articles by van der Kruit and Searle. The data are assembled in Table 3 of the paper (van der Kruit \& Searle 1982). For 7 galaxies, 4 of which enter the RFGC (NGC4244=RFGC2245; NGC5907=RFGC2946; NGC4565=RFGC2335; NGC5023=RFGC2495), the ratio $l_{0} / L_{0}$ ranges from 0.10 to 0.15 , which corresponds to $\Delta \gamma=(-4 \div-6)$. $10^{-6} \mathrm{~s} \mathrm{~km}^{-1}$.

This effect can be responsible for the observed shift, but only partially. Thus, we still need to find the reason behind the main share of the shift.

\section{SHIFT OF $\gamma$ DUE TO THE INFLUENCE OF MEASUREMENT ERRORS}

Let us show that the observed shift can be explained with purely statistical effects due to measurement errors of $\mathrm{H}$ I line widths and angular diameters.

\subsection{Estimating the impact of measurement errors: a simple case}

Before trying to address this problem at its full extent, let us consider a simple case when an analytical solution can be provided. Let us start off from introducing a toy model $y=A x+B x^{2}$, where $x=W / a$ is the main term of the Tully-Fisher relation $16, y=V$, $A=C_{1}$, and $B=\gamma_{0} C_{1}^{2}$. For generality we consider not only the value of $\gamma_{0}$ given by equation (10) but any fixed value. This model corresponds to an isotropic Hubble expansion with cosmological acceleration.

Observational data provide us with a set of $N$ points characterized by values $x_{i}$ and $y_{i}$. It is important to realize how measurement errors and deviations from the Tully-Fisher relation distort the dataset. The errors in velocity measurements and deviations from Tully-Fisher relation yield errors in $y$. The values of $A$ and $B$ determined by the least square method from the dataset with such errors will have normal distribution of errors without shift. At the same time, errors in measurements of $W$ or $a$ yield errors in $x$. This case is similar to Malmquist bias. Due to this effect, the perceived values 
of $A$ and $B$ given by the least square method will have a systematic error.

Let us consider the following case: we have $N$ values of $x_{i}$ distributed uniformly over the interval $[0,1]$ with a step $(N-1)^{-1}$. The values of $y$ are calculated with $A=A_{0}=1$ and $B=B_{0}=$ $\gamma_{0}$. In each of $N$ points the $x_{i}$ is shifted by $\sigma \xi_{i}$, where $\xi_{i}$ is a normally distributed quantity with zero mean and unit variance. The different values of $\xi_{i}$ are not correlated with each other. The values $y_{i}$ are calculated from the original nondisplaced values of $x_{i}$. The values of $A$ and $B$ given by the least square method have the form

$A=\frac{\sum_{i=1}^{N} y_{i} x_{i} \sum_{i=1}^{N} x_{i}^{4}-\sum_{i=1}^{N} y_{i} x_{i}^{2} \sum_{i=1}^{N} x_{i}^{3}}{\sum_{i=1}^{N} x_{i}^{2} \sum_{i=1}^{N} x_{i}^{4}-\left(\sum_{i=1}^{N} x_{i}^{3}\right)^{2}}$,

$B=\frac{\sum_{i=1}^{N} x_{i}^{2} y_{i} \sum_{i=1}^{N} x_{i}^{2}-\sum_{i=1}^{N} x_{i}^{3} \sum_{i=1}^{N} x_{i} y_{i}}{\sum_{i=1}^{N} x_{i}^{2} \sum_{i=1}^{N} x_{i}^{4}-\left(\sum_{i=1}^{N} x_{i}^{3}\right)^{2}}$,

$x_{i}^{0}=\frac{i-1}{N-1}, x_{i}=x_{i}^{0}+\sigma \xi_{i}, y_{i}=x_{i}^{0}+\gamma_{0}\left(x_{i}^{0}\right)^{2}$.

It is not very difficult to calculate the mean values of $A$ and $B$ over $\xi$ using the following expressions:

$A=A_{1}+\gamma_{0} A_{2}$,
$B=B_{1}+\gamma_{0} B_{2}$.

Here we designated

$A_{1}=\frac{1+40 \sigma^{2}+60 \sigma^{4}}{1+28 \sigma^{2}+180 \sigma^{4}+720 \sigma^{6}}$,

$A_{2}=\frac{28 \sigma^{2}+60 \sigma^{4}}{1+28 \sigma^{2}+180 \sigma^{4}+720 \sigma^{6}}$,

$B_{1}=\frac{-20 \sigma^{2}+120 \sigma^{4}}{1+28 \sigma^{2}+180 \sigma^{4}+720 \sigma^{6}}$,

$B_{2}=\frac{1-43 / 3 \sigma^{2}+80 \sigma^{4}}{1+28 \sigma^{2}+180 \sigma^{4}+720 \sigma^{6}}$.

Note that the formulae $32,33,34,35$ are precise up to $\mathcal{O}(1 / N)$. To verify these formulae we calculated $A$ and $B$ for this toy model using 10000 Monte Carlo simulations. The mean values perfectly fitted the given formulae (see Figure 1).

If we calculate $\gamma$ using the formula $\gamma=B / A^{2}$, we will obtain instead of true value $\gamma_{0}$ a value $\gamma$, plotted on Figure 2 against $\sigma$ for different $\gamma_{0}$. At $\sigma=0$, i.e. when there are no errors, we obtain $\gamma=\gamma_{0}$, but at small $\sigma$ we obtain $\gamma<\gamma_{0}$. This is the impact of the measurement errors we demonstrate.

\subsection{Estimating the impact of measurement errors: a real case}

The actual case is much more complicated. The problem can not be reduced to the one-dimensional case, since the galaxy's velocity depends on its position on the celestial sphere. To estimate the distance we use all the terms in the relation (16). The errors in determination of distances are non-Gaussian. They are due to the errors in angular diameters and $\mathrm{HI}$ line widths and deviations from the Tully-Fisher relation. These errors were analyzed in the paper (Parnovsky \& Parnowski 2008). Here we will briefly mention the main points of the routine used. These errors can be described by four parameters:

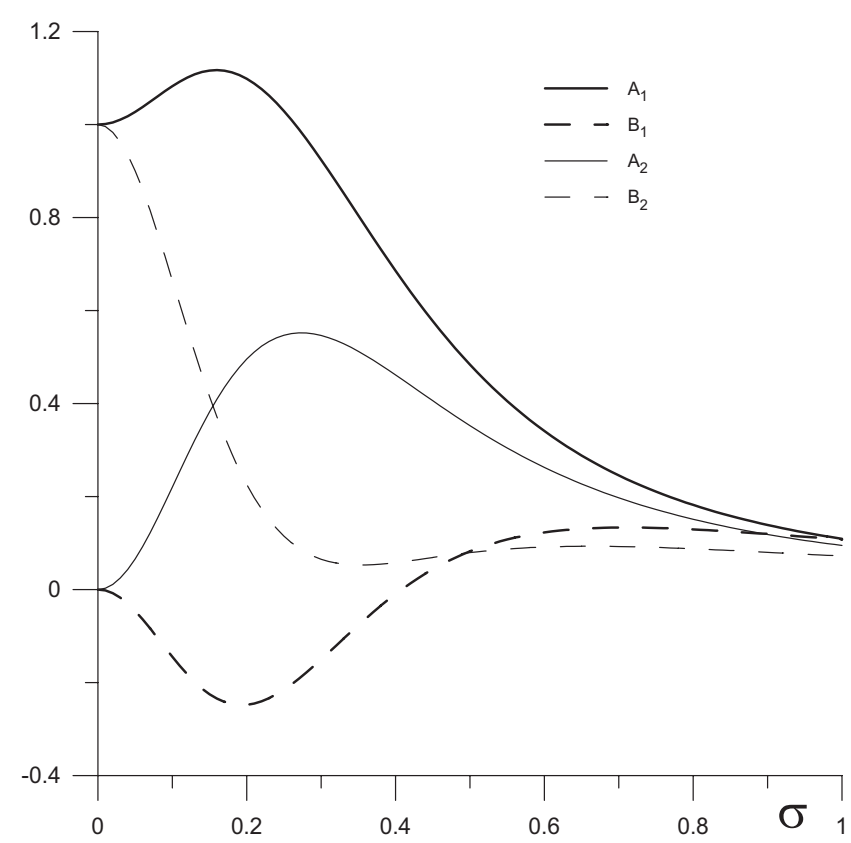

Figure 1. Dependence of the coefficients of equations (30) and 31) on the noise level

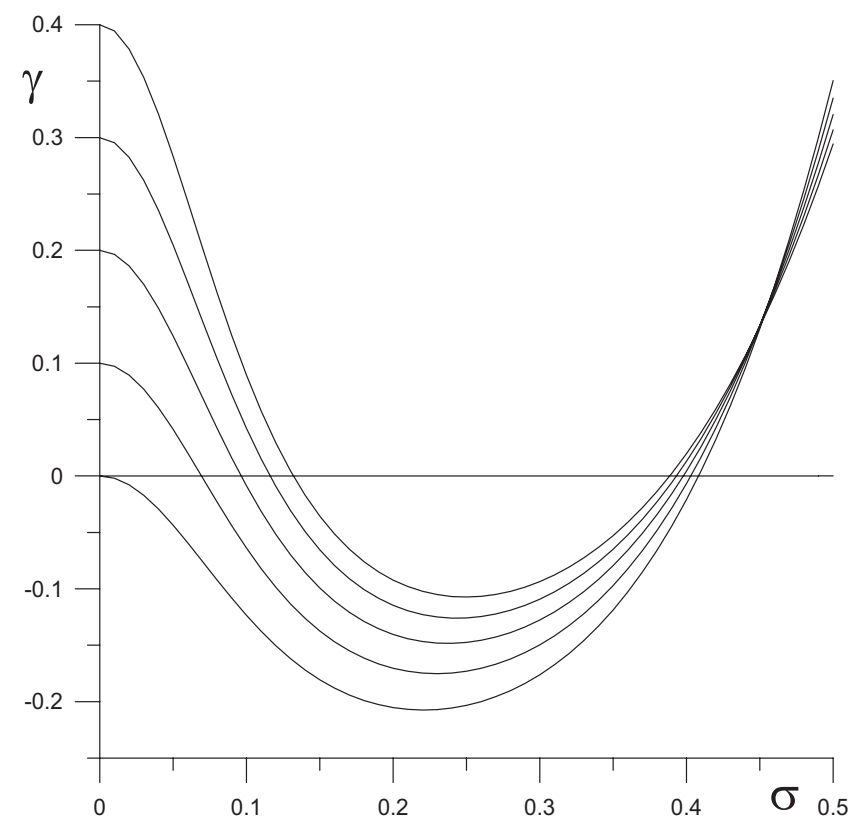

Figure 2. Dependence of least squares estimation of $\gamma$ on the noise level for different values of $\gamma_{0}$. The values of $\gamma_{0}$ equal to values of $\gamma$ at $\sigma=0$

$$
\begin{aligned}
& V_{i}=V_{i}^{(0)}\left(1+s_{V} \xi_{1}\right), \\
& W_{i}=W_{i}^{(0)}\left(1+s_{W} \xi_{2}\right), \\
& a_{i}=a_{i}^{(0)}\left(1+s_{a} \xi_{3}\right)+\Delta_{a} \xi_{4} .
\end{aligned}
$$

Here $\xi_{1}, \xi_{2}, \xi_{3}$, and $\xi_{4}$ are the four independent non-correlating random values. They are distributed according to Gauss law with zero mean and unit variance.

Let us describe what errors correspond to each type of noise. The noise (36) describes the deviations from the Tully-Fisher relationship. It does not include velocity measurement error, because 
radial velocities are well determined, as well as the directions towards galaxies. Such a type of noise provides a conventional lognormal distribution of the velocity deviation. If this noise is used alone, the maximal value of $s_{V}$ can be assumed about 0.2 , which corresponds to 20 per cent uncertainty of distance estimated by the Tully-Fisher relation.

The noise 37) corresponds to $\mathrm{H}$ I line width measurement errors. Different methods of calculating $\mathrm{H}$ I half-width of the same profile can differ by up to 10 per cent. Thus, if this noise is used alone, the maximal value of $s_{W}$ can be set to 0.15 with some tolerance.

The noise (38) describes angular diameter measurement errors. The value $s_{a}$ describes a relative error, caused by variations of exposition, curvature of galaxies etc. The diameter measurements have an error of about $5 \div 10$ per cent (Karachentseva, private communication). The value $\Delta_{a}$ describes an absolute error of measurement. This value is important for the smallest galaxies. Since these diameters were measured in tenths of millimetre, which corresponded to 0.11 arcmin, the value of $\Delta_{a}$ can be estimated between 0.05 and 0.1 . Note that if we use non-zero values of $s_{a}$ and $\Delta_{a}$ simultaneously, the maximum estimation of $\Delta_{a}$ should be somewhat reduced to avoid overestimating the noise added to angular diameters for the smallest galaxies.

Naturally, such a difficult problem of determining the shift of $\gamma$ can not be solved analytically. We use Monte Carlo simulations to resolve it numerically. Let us describe the details of this procedure.

At first, we use a subsample with $R_{\max }=10000 \mathrm{~km} \mathrm{~s}^{-1}$, which contains $N=1459$ galaxies. For each of the galaxies we substitute the measured radial velocity with the radial velocity calculated using the formulae $2,3,4,11,16$. We use the values given by equations 8 and 9 .

After that we add noise to our model and for each of 10000 simulation we calculate the coefficients in the same way as we treat actual data, i.e. using the semirelativistic model. Thus, for each realisation we obtain a complete set of the coefficients including $\gamma$. For the obtained values of $\gamma$ we calculate the mean and the standard deviation. Taking into account that the distribution of $\gamma$ is nonGaussian, its quantiles differ from the ones calculated from the normal distribution. In this article we give errors corresponding to the 99 per cent confidence level. Application of Monte Carlo method allows to do this in a straightforward way. From 10000 values of $\gamma$ for different realizations we find the 50th largest and smallest values. They give us the boundaries of the 99 per cent confidence interval.

In principle, we can apply this procedure to any mock catalogue. However, we should use a catalogue that has the same spatial distribution as well as distribution of morphological types, surface brightness index etc. as the main sample. The best mock catalogue is thus the sample itself. We use as a result the sample of actually measured parameters, namely angular diameter, surface brightness index, Hubble type, H I line width, ratio of angular diameters in red and blue imprints, and celestial coordinates. The radial velocity is calculated from the formulae (7, 16) with coefficients obtained from the real sample. Hwever, we use a more refined procedure to improve reliability.

At first we calculated the coefficients for the semirelativistic model using real data while fixing the value of $\gamma$ at $\gamma_{0}$ (10). The corresponding coefficients are also given in Table 1 Then we used this set of coefficients to calculate mock radial velocities for the galaxies. Such mock radial velocities are closer to the actual radial velocities than for any other models with fixed $\gamma$.
The values obtained using the Monte Carlo method for different parameters of the noise are given in Table 2. The top part of Table 2 illustrates the impact of each individual type of noise. One can see that the $\gamma$ value acts in the same way as in the simple case considered in the previous section. The noise $s_{V}$, corresponding to the deviations from the statistical Tully-Fisher relation has little or no effect on the value of $\gamma$. All other types of noise lead to a drastic reduction of $\gamma$, especially the noise $\Delta_{a}$. The bottom part of Table 2 contains the results obtained with realistic noise parameters. When choosing parameters we used as a control parameter the standard deviation $\sigma$ for the noised sample, comparing it to the corresponding value for real data, given in Table 1 This parameter is convenient because it vanishes in the absence of the noise and grows when the noise increases. Thus, it can help avoiding undernoising and overnoising. Other constraints used and details of the procedure are described in the paper (Parnovsky \& Parnowski 2008). Naturally, we do not try to find a unique set of noise parameters. The four noise values form a four-dimensional parameter space, the $\sigma$ constraint yields a three-dimensional hypersurface in it. Other constraints give us rough estimates of the boundaries of the volume of suitable values.

One can see from Table 2 that for a set of parameters lying inside or close to those boundaries, the value of $\gamma$ is much less than the initial value $\gamma_{0}$. Comparing these values with the value $\gamma=(-16.6 \pm 2.6) \cdot 10^{-6} \mathrm{~s} \mathrm{~km}^{-1}$ obtained from the real data, we can select a range of suitable noise parameters. For many realistic noise parameters the value of $\gamma$ falls into the $1 \sigma$ area, and for most of them it falls into 99 per cent confidence area. In those cases when $\gamma$ misses the 99 per cent confidence area, the value of $\Delta_{a}$ is unrealistically large.

We applied the same routine to the subsample with $R_{\max }=$ $8000 \mathrm{~km} \mathrm{~s}^{-1}$. The results for this subsample are also presented in Table 2 One can see that there are sets of noise parameters, which provide suitable shift of $\gamma$ for both subsamples. Nevertheless, we should mention that the same parameters provide a much less suitable shift of $\gamma$ for the subsample with $R_{\max }=6000 \mathrm{~km} \mathrm{~s}^{-1}$, where the observed value is $\gamma=(-11.0 \pm 7.6) \cdot 10^{-6} \mathrm{~s} \mathrm{~km}^{-1}$. This is due to large errors in determination of $\gamma$ for subsamples with low depth.

An additional advantage of this method is that by slight modification of the algorithm we can also estimate the influence of selection described in section 3.2 For this purpose one should add an additional condition when adding noise to angular diameters: if the noised angular diameter becomes less than the minimal angular diameter in the actual sample, the noise should be reapplied. This procedure reduces the shift of $\gamma$, but this effect is not very strong, e.g. for $R_{\max }=10000 \mathrm{~km} \mathrm{~s}^{-1}$ it gives $\gamma=(-15.6 \pm 2.4)$. $10^{-6} \mathrm{~s} \mathrm{~km}^{-1}$ against $\gamma=(-16.8 \pm 2.3) \cdot 10^{-6} \mathrm{~s} \mathrm{~km}^{-1}$. For other subsamples this effect has the same order. The best noise parameters for this case are given in the bottom part of Table 2

\section{ERRORS IN DETERMINATION OF DISTANCE CAUSED BY SHIFT OF $\gamma$}

An important result of shift of $\gamma$ is that the distances calculated by the relativistic Tully-Fisher relation $(16)$ become more than the correct ones. Really, since the combination $R+\gamma R^{2}$ is fixed by redshift data, the decrease of $\gamma$ leads to increase of $R$. We made some Monte Carlo simulations which showed that this increase is about 18 per cent. Such large errors will yield too large errors in determination of peculiar velocities, defined in a standard man- 
Table 2. Results of Monte Carlo simulations, $3 \sigma$ CL stands for 99 per cent confidence interval

\begin{tabular}{|c|c|c|c|c|c|c|c|c|c|}
\hline \multirow{3}{*}{$s_{V}$} & \multirow{3}{*}{$s_{W}$} & \multirow{3}{*}{$s_{a}$} & \multirow{3}{*}{$\Delta_{a}$} & \multicolumn{3}{|c|}{$R_{\max }=10000 \mathrm{~km} \mathrm{~s}^{-1}$} & \multicolumn{3}{|c|}{$R_{\max }=8000 \mathrm{~km} \mathrm{~s}^{-1}$} \\
\hline & & & & \multirow{2}{*}{$\sigma, \mathrm{km} \mathrm{s}^{-1}$} & \multicolumn{2}{|c|}{$\gamma, 10^{-6} \mathrm{~s} \mathrm{~km}^{-1}$} & \multirow{2}{*}{$\sigma, \mathrm{km} \mathrm{s}^{-1}$} & \multicolumn{2}{|c|}{$\gamma, 10^{-6} \mathrm{~s} \mathrm{~km}^{-1}$} \\
\hline & & & & & $\langle\gamma\rangle \pm \sigma_{\gamma}$ & $3 \sigma \mathrm{CL}$ & & $\langle\gamma\rangle \pm \sigma_{\gamma}$ & $3 \sigma \mathrm{CL}$ \\
\hline \multicolumn{10}{|c|}{ Without correction for selection } \\
\hline 0.05 & 0.00 & 0.00 & 0.00 & $282 \pm 7$ & $4.0 \pm 1.2$ & $(\quad 1.1$ & $245 \pm 6$ & $4.0 \pm 1.5$ & $(-0.9$ \\
\hline 0.10 & 00 & 0.00 & & & $4.1 \pm 2.4$ & $(-1.8$ & \pm 13 & $4.1 \pm 3.0$ & $(-6.7$ \\
\hline 0.15 & 0.00 & 0.00 & 0 & $5 \pm 21$ & $4.2 \pm 3.6$ & $(-4.1$ & \pm 19 & $4.3 \pm 4.4$ & $(-12.8$ \\
\hline 0.20 & 0.00 & 0.00 & 0.0 & $1126 \pm 28$ & $4.4 \pm 4.8$ & $(-6.7$ & & $4.6 \pm 5.9$ & $(-15.8$ \\
\hline 0.00 & 0.05 & 0.00 & & & $-0.6 \pm 1.1$ & $\left(\begin{array}{rr}-3.4 ; & 2.5)\end{array}\right.$ & & $-3.2 \pm 1.3$ & $\left(\begin{array}{rr}-8.0 ; & 2.4\end{array}\right)$ \\
\hline 0.00 & 0.1 & 0.00 & & & $-9.4 \pm 1.5$ & $(-12.8 ;-5.2)$ & & $-15.7 \pm 1.6$ & $(-20.5 ;-8.6)$ \\
\hline 0.00 & & 0. & & & $-16.5 \pm 1.3$ & $(-19.4 ;-12.8)$ & & $-24.5 \pm 1.2$ & $(-28.2 ;-18.8)$ \\
\hline 0.00 & .20 & 0.00 & & 23 & $-20.9 \pm 1.0$ & $(-23.3 ;-17.9)$ & \pm 21 & $-29.4 \pm 0.9$ & $(-32.6 ;-25.2)$ \\
\hline 0.00 & .00 & 0.05 & & & $-2.2 \pm 1.0$ & $\left(\begin{array}{ll}-4.8 ; & 0.5\end{array}\right)$ & $247 \pm 6$ & $-4.4 \pm 1.2$ & $\left(\begin{array}{cc}-9.9 ; & 0.0\end{array}\right)$ \\
\hline 0.00 & 0.00 & 0.10 & 0.00 & $38 \pm 13$ & $-14.4 \pm 1.2$ & $(-17.2 ;-11.3)$ & $468 \pm 11$ & $-19.8 \pm 1.3$ & $(-24.2 ;-14.2)$ \\
\hline 0.00 & 0.00 & 0.15 & 0.00 & $756 \pm 17$ & $-23.5 \pm 0.8$ & $(-25.4 ;-21.3)$ & $648 \pm 15$ & $-30.2 \pm 0.8$ & $(-32.7 ;-25.8)$ \\
\hline 0.00 & 0.00 & 0.20 & 0.00 & $946 \pm 23$ & $-27.3 \pm 1.0$ & $(-29.1 ;-23.3)$ & $802 \pm 21$ & $-33.8 \pm 1.0$ & $(-36.2 ;-21.6)$ \\
\hline 0.00 & 0.00 & 0.00 & 0.05 & $370 \pm 11$ & $-8.2 \pm 1.4$ & $(-12.0 ;-4.6)$ & $307 \pm 10$ & $-10.4 \pm 1.6$ & $(-16.9 ;-4.3)$ \\
\hline 0.00 & 0.00 & 0.00 & 0.10 & $664 \pm 18$ & $-21.9 \pm 1.2$ & $(-24.3 ;-16.7)$ & $548 \pm 15$ & $-27.2 \pm 1.2$ & $(-31.3 ;-1.0)$ \\
\hline 0.17 & & & & & $-17.0 \pm 2.2$ & $(-23.6 ;-5.9)$ & & $-22.6 \pm 2.4$ & $(-30.5 ;-11.4)$ \\
\hline 0.17 & & & & & $-16.0 \pm 2.3$ & $(-23.3 ;-3.9)$ & & $-21.1 \pm 2.7$ & $(-28.8 ;-9.1)$ \\
\hline 0.17 & & & & & $-16.8 \pm 2.2$ & $(-23.7 ;-6.0)$ & & $-22.2 \pm 2.6$ & $(-29.8 ;-11.2$ \\
\hline 0.18 & & & & & $-18.8 \pm 2.1$ & $(-24.9 ;-8.8)$ & & $-23.6 \pm 2.4$ & $(-31.5 ;-13.0)$ \\
\hline 0.18 & & 0.02 & & 29 & $-16.8 \pm 2.3$ & $(-23.5 ;-5.4)$ & \pm 26 & $-21.5 \pm 2.7$ & $(-30.0 ;-9.7)$ \\
\hline 0.18 & 0.05 & 0.03 & & $1174 \pm 29$ & $-17.2 \pm 2.3$ & $(-24.8 ;-6.3)$ & $1016 \pm 26$ & $-22.0 \pm 2.7$ & $(-30.0 ;-10.3)$ \\
\hline 0.18 & 0.05 & 0.04 & 0.07 & $1181 \pm 29$ & $-17.7 \pm 2.2$ & $(-24.9 ;-5.9)$ & $1022 \pm 26$ & $-22.8 \pm 2.6$ & $(-30.1 ;-11.1)$ \\
\hline \multicolumn{10}{|c|}{ With correction for selection } \\
\hline 0.18 & 0.05 & 0.02 & 0.0 & & $-15.6 \pm 2.4$ & $(-23.9 ;-4.9)$ & $1009 \pm 26$ & $-20.5 \pm 2.8$ & $(-29.0 ;-12.1$ \\
\hline 0.18 & & 0.03 & & & $-16.0 \pm 2.4$ & $(-24.1 ;-5.2)$ & $1014 \pm 26$ & $-21.0 \pm 2.7$ & $(-29.2 ;-13.0$ \\
\hline 0.18 & 0.05 & 0.04 & 0.07 & $1180 \pm 29$ & $-16.6 \pm 2.3$ & $(-24.0 ;-5.8)$ & $1020 \pm 26$ & $-21.7 \pm 2.6$ & $(-30.5 ;-13$. \\
\hline
\end{tabular}

ner $V_{p e c}=V-R$. This is a great drawback of relativistic and semirelativistic models. Note that after switching to curved spacetime, it is possible to introduce an alternative definition of a peculiar velocity $V_{\text {pec }}=V-V_{\text {cosm }}$, where $V_{\text {cosm }}$ is the velocity of cosmological expansion, which is defined in the low- $z$ limit as $V_{\text {cosm }}=R+\gamma R^{2}$. Peculiar velocities defined in this way suffer much less from this effect. Indeed, the non-relativistic model implicitly uses this second definition of peculiar velocity. If we take a look at the generalized Tully-Fisher relation (6) we will see that the term with $C_{5}$ is, in fact, quadratic in distance. Thus, the 18 per cent difference in distances leads to underestimation of the quadrupole components by a factor of 1.18 , and of the octopole one - by a factor of $(1.18)^{2}$. The dipole component remains unaltered.

To correctly determine distances in relativistic or semirelativistic models we have two options. The first one is to introduce a correction for this effect. The second, technically easier, is to eliminate the origin of this effect by fixing the value of $\gamma$ at $\gamma_{0}$. In this way we use the information about cosmological constants obtained by other more precise methods. Thus we naturally switch to the next stage - the semirelativistic model with fixed value of $\gamma$. Its 23 free parameters are given in Table 1 We also performed Monte Carlo simulations with the same noise parameters in this model. The difference between actual and estimated distances appeared to be about 0.25 per cent. Naturally, the same procedure can be applied to relativistic DQ- and D-models. In the next section we consider the collective velocity field obtained in the framework of this model of galaxy motion.

\section{THE MULTIPOLE STRUCTURE OF THE VELOCITY FIELD}

In this section we analyze the multipole structure of the velocity field. Nevertheless, we start from comparing the coefficients $C_{i}$ of the generalized Tully-Fisher relation to that obtained earlier by Parnovsky \& Gaydamaka (2004). They changed not very significantly; one should note the decrease of the coefficient corresponding to the morphological type of the galaxy and a slight increase of the coefficient corresponding to the blue diameter. However, these trends are also present in the non-relativistic model and are caused by slightly different statistical properties of the updated sample. It is also interesting that the difference of the main coefficients $C_{1}$ for the semirelativistic models with fixed $\gamma$ and with free $\gamma$ appeared to be 22 per cent, which is consistent with the 18 per cent difference in distances for these two models and is naturally caused by the same reasons.

For each regressor we calculated not only the coefficient and its error but also its statistical significance according to Fisher test. For the semirelativistic model with fixed $\gamma$ for the subsample with $R_{\max }=10000 \mathrm{~km} \mathrm{~s}^{-1}$ the minimum value $F=11.5$ corresponds to $C_{1}$, the maximum value $F=109.7-$ to $C_{2}$. These values should be compared to the values $3.8,6.6,7.9,10.8$ and 12.1, which correspond to $95,99,99.5,99.9$ and 99.95 per cent confidence levels respectively. Thus, all the coefficients of the generalised Tully-Fisher relation (16) are statistically significant at the 99.9 per cent confidence level. 


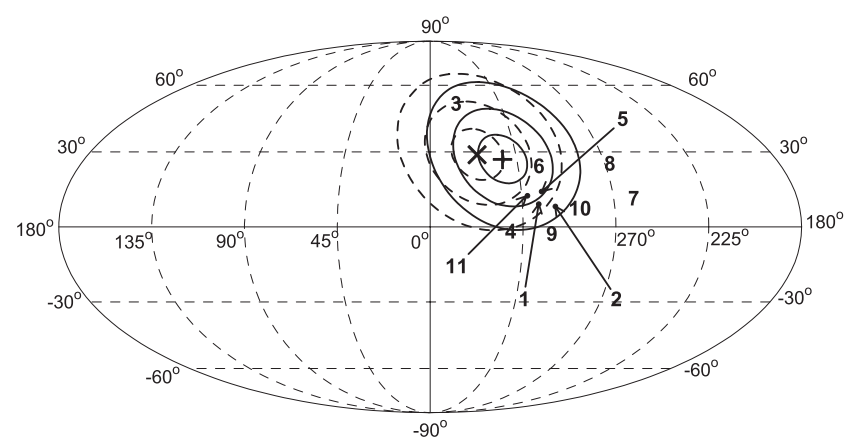

Figure 3. Bulk motion apices in galactic coordinates (Mollweide projection) for $R_{\max }=10000 \mathrm{~km} \mathrm{~s}^{-1}$. Crosses mark the apices of the bulk motion in the D-model surrounded by $1 \sigma, 2 \sigma$ and $3 \sigma$ confidence areas. Solid boundaries correspond to results in the relativistic D-model with fixed $\gamma$, and dashed ones - to the results of the nonrelativistic D-model (Parnovsky \& Parnowski 2010). Numbers denote the results of other authors: 1 - (Lynden-Bell et al. 1988), 2 - (Hudson et al. 1995), 3 - (Lauer \& Postman 1994), 4 - (Parnovsky et al. 2001), 5 (Dekel et al. 1999), 6 - (da Costa et al. 2000), 7 - (Hudson et al. 2004), 8 (Dale et al. 1999), 9 - (Kudrya et al. 2003), 10 - (Watkins et al. 2009), 11 - (Parnovsky \& Tugay 2004)

Now let us consider the dipole component of the velocity field. Its parameters including the galactic coordinates $l, b$ of the apex for the DQO-model are given in Table 1 The norms of the dipolar component do not contradict the $\Lambda \mathrm{CDM}$ model. For the model with fixed $\gamma$ the module of the dipolar component drops to $180 \mathrm{~km} \mathrm{~s}^{-1}$. However, the bulk motion is usually considered in the framework of the simplest dipole models when the only characteristics of the velocity field are the modulus and the apex of the dipole component. In our case of DQO-models the velocity field is more complex and we cannot attribute the bulk motion solely to the dipole component. For this reason, to compare our results to the results of other authors we also calculated the dipole component in the framework of the relativistic (the same as semirelativistic) D-model with fixed $\gamma$. It yields the bulk flow velocity of $314 \mathrm{~km} \mathrm{~s}^{-1}$ directed towards $l=322^{\circ}, b=27^{\circ}$ (Centaurus). On Figure 3 we plotted the boundaries of $1 \sigma, 2 \sigma$ and $3 \sigma$ confidence areas of this apex for $R_{\max }=10000 \mathrm{~km} \mathrm{~s}^{-1}$. For this purpose we projected the 8-dimensional ellipsoid of errors into the 3-dimensional space and then projected it on the celestial sphere. On the same figure we also plotted the boundaries of the confidence areas of the apex in non-relativistic D-model (Parnovsky \& Parnowski 2010) as well as positions of apices obtained by different authors. The value of the bulk motion appears to be larger than for DQO-models. For the subsample with $R_{\text {max }}=8000 \mathrm{~km} \mathrm{~s}^{-1}$ it is equal to $285 \mathrm{~km} \mathrm{~s}^{-1}$. We see that D-models provide a result, which is closer to that obtained by Watkins et al. (2009), but still consistent with the $\Lambda$ CDM model.

Let us now consider the quadrupole component of the velocity field. What is the physical sense of the quadrupole component? As one can see from the paper (Parnovsky et al. 2001), it can be naturally combined with the Hubble constant. As a result, we obtain the effective 'Hubble constant' depending on direction

$H(l, b)=H\left(1+Q_{i k} n_{i} n_{k}\right)$.

Naturally, this effective 'Hubble constant' is caused by the largescale collective motion on the sample scale. To estimate the value of its anisotropy we found the eigenvalues and eigenvectors of ten- sor $Q$. The three eigenvectors are orthogonal and the sum of three eigenvalues is equal to zero because $\mathbf{Q}$ is a traceless tensor.

We found the eigenvalues and the eigenvectors of the tensor $\mathbf{Q}$ for two considered subsamples. For the subsample with $R_{\max }=10000 \mathrm{~km} \mathrm{~s}^{-1}$ the maximal eigenvalue $7.1 \pm 1.7$ per cent corresponds to an axis directed towards $l=98^{\circ}, b=79^{\circ}$ (Canes Venatici) and the opposite direction (Phoenix). The minimal eigenvalue $-4.1 \pm 1.7$ per cent corresponds to an axis directed towards $l=195^{\circ}, b=2^{\circ}$ (Gemini) and the opposite direction (Sagittarius). The third eigenvalue -3 per cent corresponds to an axis directed towards $l=286^{\circ}, b=11^{\circ}$ (Centaurus-Vela) and the opposite direction (Andromeda-Lacerta). Comparing these values to the non-relativistic model (Parnovsky \& Parnowski 2010) one can see that both the eigenvalues and the directions of the axes changed insignificantly. Nevertheless, the two negative eigenvalues, which are close to each other, have the opposite order in these two models. In this sense, the positive axis notably stands out, for which the effective 'Hubble constant' exceeds the mean value by 7 per cent. For the subsample with $R_{\max }=8000 \mathrm{~km} \mathrm{~s}^{-1}$ the ellipsoid is threeaxial and essentially differs from the oblate spheroid. The maximal eigenvalue $7.1 \pm 1.7$ per cent corresponds to an axis directed towards $l=102^{\circ}, b=85^{\circ}$ (Canes Venatici) and the opposite direction (Sculptor). The minimal eigenvalue $-5.3 \pm 1.6$ per cent corresponds to an axis directed towards $l=266^{\circ}, b=5^{\circ}$ (Vela) and the opposite direction (Cygnus). The third eigenvalue -2 per cent corresponds to an axis directed towards $l=356^{\circ}, b=1^{\circ}$ (SagittariusScorpio) and the opposite direction (Auriga-Taurus). These values are very close to those given by the non-relativistic model. Note that the axes for both subsamples nearly coincide with the exception of reverse order of negative eigenvalues for $R_{\max }=10000 \mathrm{~km} \mathrm{~s}^{-1}$.

We also calculated the statistical significance of these eigenvalues. For $R_{\max }=10000 \mathrm{~km} \mathrm{~s}^{-1}$ the maximal eigenvalue has $F=18.2$, which means that it is non-zero at 99.95 per cent confidence level, and the minimal eigenvalue has $F=5.8$, which means that it is non-zero at 97.5 per cent confidence level. The similar situation holds for the subsample with $R_{\max }=8000 \mathrm{~km} \mathrm{~s}^{-1}$ with Fisher values being 18.3 and 10.7 respectively. Additionally, we calculated the total statistical significance of the quadrupole component. The value $V^{q u a}$ with its 5 degrees of freedom appears to be non-zero at over 99.5 per cent confidence level according to F-test.

In the same way we calculated the total statistical significance of the octopole component. The value $V^{\text {oct }}$ with its 10 degrees of freedom appears to be non-zero at over 99.5 per cent confidence level according to F-test. The value $\boldsymbol{P}$ with its 3 degrees of freedom appears to be non-zero at slightly less than 90 per cent confidence level according to F-test. Unlike the quadrupole component, the octopole one lacks easily interpretable characteristics like eigenvector apices. The radial velocity field for $R=8000 \mathrm{~km} \mathrm{~s}^{-1}$ and $R_{\max }=10000 \mathrm{~km} \mathrm{~s}^{-1}$ in the semirelativistic model, which includes the octopole component, appeared to be very similar to that in non-relativistic case, depicted on Fig. 6 in the article (Parnovsky \& Parnowski 2010). The most prominent feature of both these velocity fields is a strong inbound flow coming from the direction opposite to the apex of the bulk flow.

Thus we obtained that the velocity field in the semirelativistic model with fixed $\gamma$ is very similar to that in non-relativistic case. The difference between these velocity fields may become significant when more precise and deep samples will be available. For the existing sample this similarity yields two conclusions. The first one is that this similarity justifies the form of the generalised TullyFisher relation for the non-relativistic model. In contrast to the relativistic model, the non-relativistic model (6) was introduced em- 
pirically. It includes the term, quadratic in distance, which has no theoretical substantiations. The relativistic model considered here explains why such a term needs to be included and what its order is. The second one is that the external information about the cosmological deceleration parameter, which we introduced into the model by fixing $\gamma$, is consistent with the observed parameters of RFGC galaxies.

The bulk flow velocity is the most sensitive characteristic to the selection of the model used. It can change as much as 1.5 times when the same data is processed with different models of motion (314 $\mathrm{km} \mathrm{s}^{-1}$ for D-model, $281 \mathrm{~km} \mathrm{~s}^{-1}$ for DQ-model, $181 \mathrm{~km} \mathrm{~s}^{-1}$ for DQO-model, and $249 \mathrm{~km} \mathrm{~s}^{-1}$ for DQO-model without vector $\boldsymbol{P}$ - all with fixed $\gamma$ for $100 h^{-1} \mathrm{Mpc}$ ). This yields two considerations. First, the bulk flow velocity is a vulnerable characteristic of collective motion for deep samples. Second, some authors like Wyman (2010) believe that the excessively large values of bulk flow velocities obtained by some authors are a sufficient reason for abandoning the $\Lambda \mathrm{CDM}$ cosmology for more exotic theories like brane cosmologies. We, however, have a different opinion on this matter, and consider that such results should be double-checked using different models of collective motion.

\section{CONCLUSION}

We applied the relativistic model of motion supplied with the generalised Tully-Fisher relation (16) to the sample of 1623 flat edgeon spiral galaxies from the RFGC catalogue. The analysis of results prompted us to switch first to the semirelativistic model, and then to the semirelativistic model with fixed $\gamma$. The parameters of the collective motion obtained in the framework of this model appeared to be close to that obtained in the non-relativistic case. We analysed certain reasons behind the decrease of $\gamma$ in the semirelativistic model. Evolution of galactic diameters, selection effects, and difference between isophotal and angular diameter distances appeared to be inadequate to explain this effect. At the same time, measurement error in $\mathrm{H}$ I line widths and angular diameters can easily provide such a decrease. This was illustrated in a toy model, which allows analytical consideration, and then in the full model using Monte Carlo simulations. The obtained bulk flow velocity is consistent with $\Lambda \mathrm{CDM}$ cosmology.

\section{REFERENCES}

da Costa L.N. et al., 2000, ApJ, 537, L81

Dale D.A. et al., 1999, ApJ, 510, L11

Dekel A. et al., 1999, ApJ, 522, 1

Fathi K. et al., 2010, preprint [arXiv:1004.1507]

Fisher R.A., 1954, "Statistical methods for research workers", Oliver and Boyd: London

Freeman K.C., 1970, ApJ, 160, 811

Hudson D.J., 1964, "Statistics Lectures on Elementary Statistics and Probability", CERN: Geneva

Hudson M.J. et al., 1995, MNRAS, 274, 305

Hudson M.J. et al., 2004, MNRAS, 352, 61

Karachentsev I.D. et al., 1993, AN, 314, 97

Karachentsev I.D. et al., 1999, Bull. SAO, 47, 5 (astro-ph/0305566)

Karachentsev I.D. et al., 2000, Astron. Rep., 44, 150

Komatsu E. et al., 2010, preprint |arXiv:1001.4538|
Kudrya Yu.N., Alexandrov A.N., 2002, J. Phys. Studies, 6, 472 (in Ukrainian)

Kudrya Yu.N., Alexandrov A.N., 2004, Herald Kyiv Univ. Astron., 39-40, 130 (in Ukrainian)

Kudrya Yu.N. et al., 1997, Astron. Lett., 23, 11

Kudrya Yu.N. et al., 2003, A\&A, 407, 889

Lauer T.R., Postman M., 1994, ApJ, 425, 418

Lynden-Bell D. et al., 1988, ApJ, 326, 19

Marinoni C. et al., 1998, ApJ, 505, 484

Parnovsky S.L., Gaydamaka O.Z., 2004, Kinematics and Physics of Celestial Bodies, 20, 477

Parnovsky S.L., Tugay A.V., 2004, Astron. Lett., 30, 357

Parnovsky S.L., Parnowski A.S., 2008, AN, 329, 864

Parnovsky S.L., Parnowski A.S., 2009, preprint [arXiv:0911.3102]

Parnovsky S.L., Parnowski A.S., 2010, Ap\&SS, 325, 163 |arXiv:0910.4640|

Parnovsky S.L. et al., 2001, Astron. Lett., 27, 765

Reshetnikov V.P. "Surface photometry of galaxies", http://www.astro.spbu.ru/staff/resh/Book/index.html (in Russian)

Sandage A., Lubin L.M., 2001, ApJ, 121, 2271

Tolman R.C., 1930, Proc. Nat. Acad. Sci., 16, 5111

Tolman R.C., 1934, "Relativity, Thermodynamics, and Cosmology", Oxford Univ. Press: Oxford, 467

van der Kruit P.C., Searle L., 1982, A\&A, 110, 61

de Vaucouleurs G., 1959, Handbuch der Physik, 53, 311

de Vaucouleurs G. et al., 1991, "Third Reference Catalogue of Bright Galaxies", Springer: Berlin, Heidelberg, New York

Watkins R. et al., 2009, MNRAS, 392, 743

Weinberg S., 1972, "Gravitation and Cosmology: Principles and Applications of the General Theory of Relativity", Wiley: New York, London, Sydney, Toronto

Wyman M., Khoury J., 2010, preprint arXiv:1004.2046

Zeldovich Ia.B., Novikov I.D., 1983, "Relativistic astrophysics", University of Chicago Press: Chicago

This paper has been typeset from a $\mathrm{T}_{\mathrm{E}} \mathrm{X} / \mathrm{LT}_{\mathrm{E}} \mathrm{X}$ file prepared by the author. 